\title{
Erratum to: Clinical experience with four cases of jackhammer esophagus
}

\author{
Rui Nakato $^{1}$ - Noriaki Manabe ${ }^{2}$ Naoshi Mitsuoka ${ }^{3} \cdot$ Hironari Shiwaku ${ }^{4}$. \\ Hideo Matsumoto ${ }^{5}$ Kanefumi Yamashita ${ }^{4}$ Akiko Shiotani $^{1} \cdot$ Jiro Hata $^{2}$. \\ Tetsuo Watanabe ${ }^{3} \cdot$ Toshihiro Hirai $^{5} \cdot$ Ken Haruma $^{6}$
}

Published online: 6 September 2017

(C) The Japan Esophageal Society and Springer Japan KK 2017

\section{Erratum to: Esophagus (2016) 13:208-214 DOI: 10.1007/s10388-015-0518-0}

In the original publication of the article, the Conflict of interest statement was published incorrectly. The correct Conflict of interest statement should be as below.

Ken Haruma received honorarium from Abbott Japan Co., Ltd. Astellas Pharma Inc., AstraZeneca plc, Daiichi Sankyo Company, Ltd., Deep Impact Co., Ltd., EA Pharma Co., Ltd., Kyorin Pharmaceutical Company, Ltd., Mitsubishi Tanabe Pharma Corporation, Otsuka Pharmaceutical Co., Ltd., Research Organization for GastroEnterological Disease Tereatment, Sumitomo Dainippon Pharma Co., Ltd., Takeda Pharmaceutical Company Ltd., Tumura \& Co., Zeria Pharmaceutical Co., Ltd. Toshihiro Hirai received

The online version of the original article can be found under doi:10.1007/s10388-015-0518-0.

Noriaki Manabe

n_manabe@hkg.odn.ne.jp

1 Division of Gastroenterology, Department of Internal Medicine, Kawasaki Medical School, Kurashiki, Japan

2 Division of Endoscopy and Ultrasonography, Department of Clinical Pathology and Laboratory Medicine, Kawasaki Medical School, 577 Matsushima, 701-0192 Kurashiki, Japan

3 Department of Gastroenterology, Watanabe Gastrointestinal Hospital, Kurashiki, Japan

4 Department of Gastroenterological Surgery, Fukuoka University Faculty of Medicine, Fukuoka, Japan

5 Department of Gastroenterological Surgery, Kawasaki Medical School, Kurashiki, Japan

6 Department of General Internal Medicine 2 Gastroenterology and Hepatology, Kawasaki Medical School, Kurashiki, Japan honorarium from Abbott Japan Co., CHUGAI Pharmaceutical Co., Ltd., Daiichi Sankyo Company, Ltd., Otsuka Pharmaceutical Co., Ltd., Otsuka Pharmaceutical Factory, Inc., SHIONOGI \& Co., Ltd., TAIHO pharmaceutical Co., Ltd., Takeda Pharmaceutical Company Ltd. Jiro Hata received honorarium from AstraZeneca plc, EA Pharma Co., Ltd., Toshiba Medical Systems Co., Tumura \& Co. Akiko Shiotani received honorarium from AbbVie GK, Astellas Pharma Inc., AstraZeneca plc, Daiichi Sankyo Company, Ltd., EA Pharma Co., Ltd., Medtronic Japan Co., Ltd., Mitsubishi Tanabe Pharma Corporation, Otsuka Pharmaceutical Co., Ltd., Research Organization for GastroEnterological Disease Tereatment, Takeda Pharmaceutical Company Ltd., Tumura \& Co., Zeria Pharmaceutical Co., Ltd. Hideo Matsumoto received honorarium from Abbott Japan Co., CHUGAI Pharmaceutical Co., Ltd., Daiichi Sankyo Company, Ltd., Otsuka Pharmaceutical Co., Ltd., Otsuka Pharmaceutical Factory, Inc., SHIONOGI \& Co., Ltd., TAIHO pharmaceutical Co., Ltd., Takeda Pharmaceutical Company Ltd. Noriaki Manabe received honorarium from Abbott Japan Co., Ltd., AstraZeneca plc, Daiichi Sankyo Company, Ltd., Takeda Pharmaceutical Company Ltd. 